\title{
ESTUDO SOBRE MALÁRIA E PARASITOSES INTESTINAIS EM INDIGENAS DA TRIBO NADËB-MAKU, ESTADO DO AMAZONAS, BRASIL
}

\author{
Odair Genaro* \\ José J. Ferraroni**
}

$\bullet$

\begin{abstract}
GENARO, O. \& FERRARONI, J. J. Estudo sobre malária e parasitoses intestinais em indígenas da tribo Nadëb-Maku, Estado do Amazonas, Brasil. Rev, Saúde públ., S. Paulo, 18: $162-9,1984$.
\end{abstract}

RESUMO: Em março de 1983 detectou-se uma epidemia de malária por Plasmodium falciparum na tribu indígena Nadéb-Maku localizada às margens do Rio Uneiuxi, alto Rio Negro, no Estado do Amazonas (Brasil). Foram obtidas e examinadas para hematozoários amostras de sangue periférico de 76 indígenas. Vinte e sete $(35,5 \%)$ dessas amostras estavam positivas para plasmódios. A infecção malárica foi tratada com Fansidar@ (pirimetamina + sulfadoxina), mefloquina e/ou primaquina. A única espécie de anofelino coletada na aldeia durante o período da epidemia foi Anopheles mediopunctatus. Amostras de fezes obtidas de 49 indígenas foram examinadas para parasitas intestinais e $100 \%$ delas estavam positivas. A maioria dos indígenas estavam parasitada por mais de uma espécie de parasita.

UNITERMOS: Plasmodium falciparum. Malária, tratamento. Indios brasileiros.

\section{INTRODUÇÃO}

Em março de 1983 fomos informados da ocorrência de febre alta nos indivíduos componentes da tribo indígena dos Nadëb-Maku. Esta tribo habita uma aldeia localizada apro. ximadamente a $01^{\circ} 12^{\prime}$ sul e $66^{\circ} 04^{\prime}$, oeste no Estado do Amazonas, às margens do Rio Uneiuxí, afluente do Rio Negro (Figura). Com a devida permissão da Fundação Nacional do Indio (FUNAI) foi imediatamente prestada assistência aos indígenas e ao mesmc tempo feito diagnóstico de saúde daquela população. A urgência de se atingir o local prendia-se ao fato de que alguns ameríndios haviam chegado a Manaus para praticar comércio, juntamente com outros caboclos. Alguns tinham problemas de saúde, com história clínica de febre há vários dias, acompanhada de cefaléia e vômitos. Dentre eles, 4 eram portadores de malária por $P$. falcipa- rum. O problema era preocupante por saber que esta área do Estado do Amazonas era considerada livre de malária. A possibilidade de existir um vetor potencial na área poderia levar ao risco de uma epidemia às populações adjacentes, embora distantes, mas de contacto devido ao comércio realizado entre os índios e caboclos da área.

Este trabalho apresenta e discute os resultados da expedição científica e em linhas gerais o diagnóstico de saúde dos habitantes da aldeia Nadëb-Maku.

\section{MATERIAL E MÉTODOS}

A população da aldeia era constituída de 79 indivíduos, incluindo adultos e crianças de ambos os sexos, habitando 12 malocas situadas próximas uma das outras, à margem direita do Rio Uneiuxí. Foram coletadas amostras de sangue de todos os indios

\footnotetext{
* Do Instituto Nacional de Pesquisas da Amazônia (INPA) - Estrada do Aleixo, 1756 69000 - Manaus, AM - Brasil.

** Do Conselho Nacional de Desenvolvimento Científico e Tecnológico (CNPq) e da Universidade Federal do Amazonas - 69000 - Manaus, AM - Brasil.
} 
GENARO, O. \& FERRARONI, J. J. Estudo sobre a malária e parasitoses intestinais em indígenas da tribo Nadëb-Maku, Estado do Amazonas, Brasil. Rev. Saúde públ., S. Paulo, 18:162 - 9, 1984.

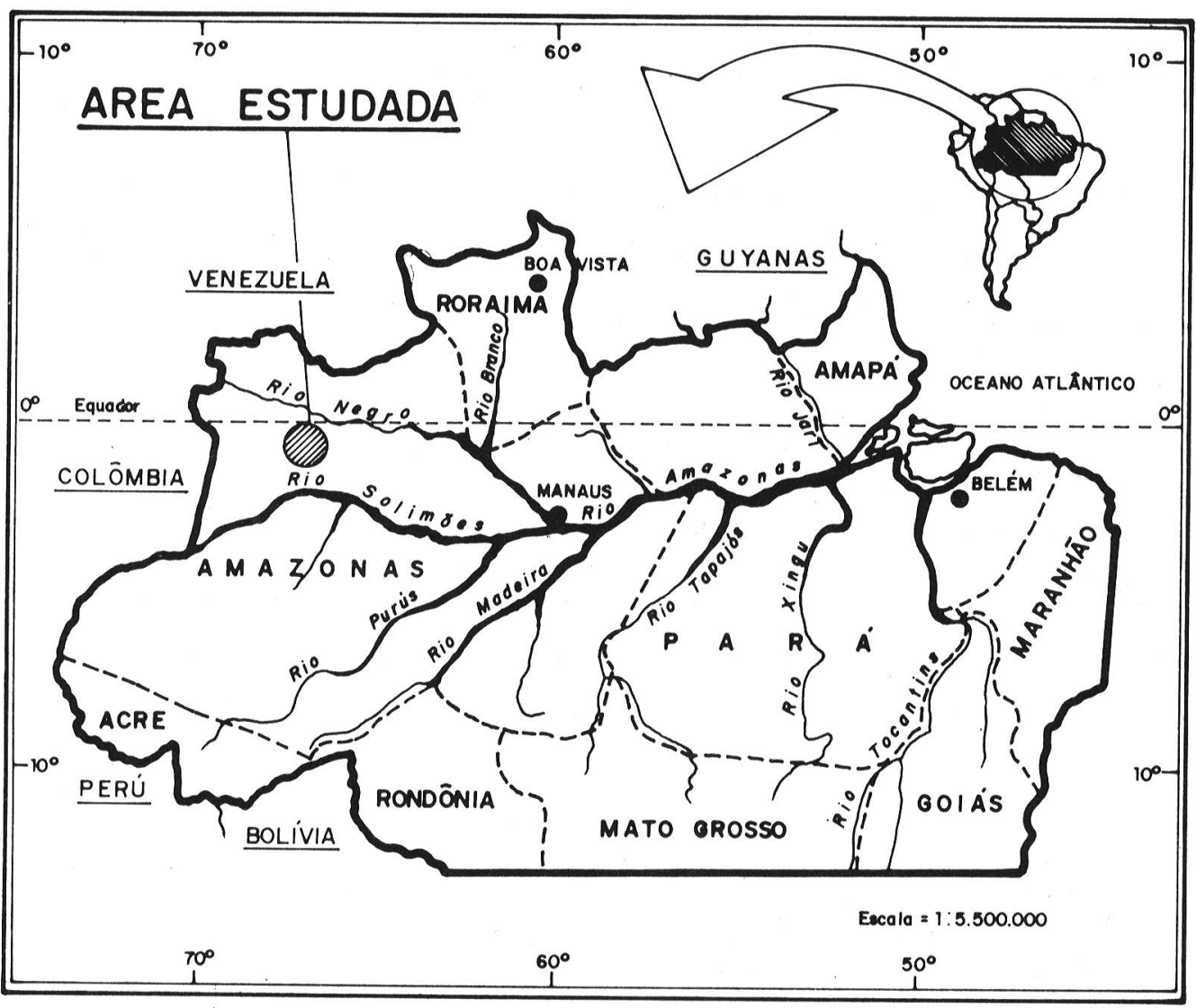

Localização geográfica da tribo NADEB-MAKU na Amazônia brasileira.

da aldeia. De cada amostra preparou-se gota espessa em lâminas coradas pelo Giemsa para pesquisa de plasmódios, seguindo os padrões clássicos recomendados pela Organização Mundial da Saúde. Todo índigena, com presença de parasitas no sangue periférico, foi tratado. As drogas utilizadas foram pirimetamina + sulfadoxina (Fansidar $\left.{ }^{\circledR}\right) 20 \mathrm{mg} / \mathrm{kg} /$ peso, dose única oral (Laboratório Roche, Rio de Janeiro); Mefloquina (Ro 21.5998/ $603) 10 \mathrm{mg} / \mathrm{kg} /$ peso, dose única oral (Laboratório Roche, Neuilly, França) e primaquina $1,0 \mathrm{mg} / \mathrm{kg} /$ peso divididos em 01 dose diária por 3 dias consecutivos, via oral. Um único paciente recebeu Fansidar ${ }^{\circledR}$ intramuscular. A parasitemia periférica foi acompanhada até $\mathbf{1 0}$ dias após o tratamento.
Foram coletados anofelinos intra e peridomiciliar durante quatro dias consecutivos obedecendo ao horário das 18:00 às 24:00 horas, mediante o uso de isca humana.

Coletou-se também amostras de fezes da maioria da população, as quais foram conservadas em Mertiolate, Iodo e Formol (MIF) para pesquisas de helmintos e protozoários. Essas fezes foram estudadas pelo método de Ritchie, considerado simples e eficiente tanto para helmintos como protozoários. Todos os indivíduos da aldeia foram tratados com anti-helmíntico polivalente.

$\mathrm{Na}$ última semana, antes de nossa chegada à área, haviam falecido quatro índios da tribo, com história clínica compatível de malária. No dia de nossa chegada à tribo, falece- 
GENARO, O. \& FERRARONI, J. J. Estudo sobre a malária e parasitoses intestinais em indígenas da tribo Nadëb-Maku, Estado do Amazonas, Brasil. Rev. Saúde públ., S. Paulo, 18:162 - 9, 1984.

\section{TABELA 1}

Prevalência de malária falciparum por grupo etário e sexo em índios da tribo Nadêb-Maku em março de 1983.

\begin{tabular}{|c|c|c|c|c|c|c|c|c|c|c|}
\hline \multirow{2}{*}{$\begin{array}{l}\text { Idade } \\
\text { (anos) }\end{array}$} & \multicolumn{5}{|c|}{ Masculino } & \multicolumn{5}{|c|}{ Feminino } \\
\hline & Positivo & $(\mathscr{\infty})$ & Negativo & (\%) & Total & Positivo & (\%) & Negativo & (\%) & Total \\
\hline $\begin{array}{r}-10 \\
11-20 \\
21-30 \\
31-40 \\
41-50 \\
50-\end{array}$ & $\begin{array}{l}7 \\
8 \\
1 \\
3 \\
1 \\
3\end{array}$ & $\begin{array}{l}(39) \\
(57) \\
(25) \\
(60) \\
(33) \\
(43)\end{array}$ & $\begin{array}{r}11 \\
6 \\
3 \\
2 \\
2 \\
4\end{array}$ & $\begin{array}{l}(61) \\
(43) \\
(75) \\
(40) \\
(67) \\
(57)\end{array}$ & $\begin{array}{r}18 \\
14 \\
4 \\
5 \\
3 \\
7\end{array}$ & $\begin{array}{l}1 \\
1 \\
2 \\
- \\
- \\
-\end{array}$ & $\begin{array}{l}(11) \\
(20) \\
(29) \\
- \\
- \\
-\end{array}$ & $\begin{array}{l}8 \\
4 \\
5 \\
1 \\
2 \\
1\end{array}$ & $\begin{array}{r}(89) \\
(80) \\
(71) \\
(100) \\
(100) \\
(100)\end{array}$ & $\begin{array}{l}9 \\
5 \\
7 \\
1 \\
2 \\
1\end{array}$ \\
\hline Total & 23 & (45) & 28 & (55) & 51 & 4 & (16) & 21 & (84) & 25 \\
\hline
\end{tabular}

ram mais 3 índios de malária falciparum. Esses índios foram a óbitos antes mesmo de iniciarem o tratamento.

\section{RESULTADOS}

Dos 76 índios examinados para malá. ria, $27(35,5 \%)$ apresentaram parasitemia periférica por $P$. falciparum. 0 grupo etário mais atingido foi o menor de 10 anos, com maior prevalência para o sexo masculino (Tabela 1). Vinte e dois individuos com parasitas no sangue periférico foram tratados e seguidos por 10 dias (ño foi possivel fazer o seguimento de cinco pacientes), dos quais 20 eram do sexo masculino e 2 feminino: 17 receberam Fansidar $(8)$ foram tratados com Mefloquina. A parasitemia periférica variou de 01 parasita por lâmina até 200 parasitas por campo em gota espêssa. Ambos os tratamentos foram eficazes, e no segundo dia de tratamento, não se encontrava parasitas no sangue periférico dos pacientes (Tabela 2). Durante as quatro noites de coleta (18:00 a $24: 00$ horas) somente 15 exemplares de anofelinos foram capturados na aldeia, no periodo entre as 19:00 e 21:00 h., e todos pertencentes à mesma espécie: Anopheles $(A)$ mediopunctatus.

Em todas as 49 amostras de fezes observouњe a presença de parasitas intestinais, com maior prevalência para helmintos, em particular ancilostomídeos, com 47 (96\%) seguidos por Trichiurus trichiura e Ascaris lumbricoides com $33(67,3 \%)$ e $30(61,2 \%)$, respectivamente. Entre os protozoários a Entamoeba histolytica foi a mais representativa com $18(36,7 \%)$ (Tabela 3). A Tabela 4 mostra o comportamento do poliparasitismo intestinal nesta população indígena. $\mathrm{E}$ interessante observar que houve indivíduos parasitados por 5,6 e até 7 espécies de parasitas. Em somente 4 indivíduos observouse um único parasita intestinal. A Tabela 5 evidencia a distribuição por grupo etário e sexo das 49 amostras de fezes desta população, com as respectivas espécies de parasitas.

\section{DISCUSSÃO}

A malária não tem afetado muito a população indígena estável da Amazônia. Isto é fácil de ser entendido pois a malária está intimamente relacionada com a mobilidade das populações, como nos projetos de assentamento dirigido, núcleos de colonização, minerações e aberturas de estradas. Os migrantes infectados, após passarem por áreas endêmicas, são verdadeiros disseminadores da infecção, mesmo porque nos indivíduos não imunes os gametócitos permanecem 
GENARO, O. \& FERRARONL, J. J. Estudo sobre a malária e parasitoses intestinais em indígenas da tribo Nadëb-Maku, Estado do Amazonas, Brasil. Rev. Saúde públ., S. Paulo, 18:162 - 9, 1984.

TABELA 2

Comportamento da parasitemia periférica em 22 indígenas Nadeb-Maku tratados para malária falciparum em março de $1983 *$.

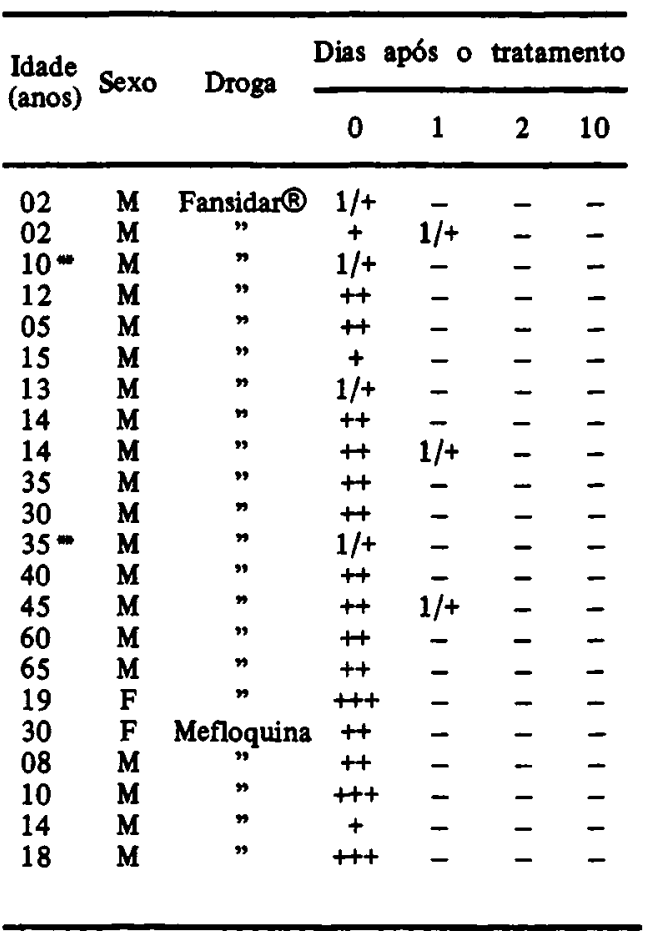

* = não foi possível seguir 5 pacientes por 10 dias

$\mathbf{M}$ = masculino

F $=$ feminino

$1 /+=$ menos de 1 parasito por campo em gota espessa.

- = ausência de parasito no sangue periférico.

* = presença unicamente de gametócitos.

$+\quad=1$ parasito por campo em gota espessa.

$+=2$ a 20 parasitos por campo em gota espessa.

$++=21$ a 200 parasitos por campo em gota espessa.
TABELA 3

Prevalência de parasitos intestinais em 49 indígenas da tribo Nadeb-Maku, Amazonas, março de 1983.

\begin{tabular}{lrr}
\hline \multicolumn{1}{c}{ Parasitos } & $\begin{array}{c}\text { Número de Prevalência } \\
\text { positivos }\end{array}$ & (\%) \\
\hline Ascaris lumbricoides & 30 & 61,2 \\
Ancilostomideos & 47 & 95,9 \\
Strongyloides stercoralts & 6 & 12,2 \\
Trichiurus trichtura & 33 & 67,3 \\
Entamoeba histolytica & 18 & 36,7 \\
Entamoeba coli & 16 & 32,6 \\
Giandia lamblia & 4 & 8,1 \\
Endolimax nana & 8 & 16,3 \\
Chilomastix mesnili & 2 & 4,0 \\
Pentatrichomonas hominis & 1 & 2,0 \\
& & \\
\hline
\end{tabular}

TABELA 4

Prevalência de poliparasitismo em 49 índios da tribo Nadeb-Maku, Amazonas, março de 1983.

\begin{tabular}{ccc}
\hline Amostras & $\begin{array}{c}\text { Espécies de } \\
\text { parasitos por } \\
\text { amostras }\end{array}$ & $\%$ \\
\hline 4 & 1 & 8,2 \\
11 & 2 & 22,4 \\
16 & 3 & 32,6 \\
8 & 4 & 16,4 \\
7 & 5 & 14,2 \\
2 & 6 & 4,1 \\
1 & 7 & 2,1 \\
\hline Total 49 & - & 100 \\
\hline
\end{tabular}


GENARO, O. \& FERRARONI, J. J. Estudo sobre a malária e parasitoses intestinais em indígenas da tribo Nadëb-Maku, Estado do Amazonas, Brasil. Rev. Saúde púbi., S. Paulo, 18:162 - 9, 1984.

\section{TABELA 5}

Distribuição por grupo etário e sexo da população Nadèb-Maku em relação aos parasitos encontrados pelo exame de fezes.

\begin{tabular}{|c|c|c|c|c|c|c|c|c|c|c|c|c|c|c|c|c|c|c|c|}
\hline \multirow{2}{*}{$\begin{array}{l}\text { Idade } \\
\text { (anos) }\end{array}$} & \multicolumn{10}{|c|}{ Masculino } & \multicolumn{9}{|c|}{ Feminino } \\
\hline & $\mathrm{Al}$ & Ad & $\mathrm{Tt}$ & Ss & Eh & $\mathrm{Gl}$ & $\mathrm{Ec}$ & En & $\mathrm{Cm}$ & $\mathrm{Ph}$ & $\mathrm{A}$ & Ad & $\mathrm{Tt}$ & Ss & Eh & $\mathrm{Gl}$ & Ec & En & $\mathrm{Cm}$ \\
\hline $\begin{array}{r}-10 \\
11-20 \\
21-30 \\
31-40 \\
41-50 \\
51-\end{array}$ & $\begin{array}{l}5 \\
5 \\
3 \\
1 \\
2 \\
3\end{array}$ & $\begin{array}{r}10 \\
6 \\
3 \\
4 \\
2 \\
5\end{array}$ & $\begin{array}{l}9 \\
7 \\
1 \\
2 \\
2 \\
2\end{array}$ & $\begin{array}{l}1 \\
2 \\
- \\
1 \\
- \\
-\end{array}$ & $\begin{array}{l}4 \\
2 \\
1 \\
1 \\
2 \\
1\end{array}$ & $\begin{array}{l}1 \\
- \\
\overline{1} \\
\overline{1}\end{array}$ & $\begin{array}{l}3 \\
6 \\
1 \\
2 \\
1 \\
2\end{array}$ & $\begin{array}{l}2 \\
\overline{1} \\
- \\
\overline{1}\end{array}$ & $\begin{array}{l}\overline{1} \\
- \\
- \\
- \\
-\end{array}$ & $\begin{array}{l}1 \\
- \\
- \\
- \\
- \\
-\end{array}$ & $\begin{array}{r}3 \\
2 \\
4 \\
2 \\
- \\
-\end{array}$ & $\begin{array}{c}7 \\
3 \\
4 \\
2 \\
1 \\
-\end{array}$ & $\begin{array}{r}4 \\
1 \\
3 \\
2 \\
- \\
-\end{array}$ & $\begin{array}{l}1 \\
- \\
- \\
- \\
-\end{array}$ & $\begin{array}{l}3 \\
- \\
2 \\
1 \\
1 \\
-\end{array}$ & $\begin{array}{l}1 \\
- \\
- \\
- \\
-\end{array}$ & $\begin{array}{l}\overline{1} \\
- \\
- \\
- \\
-\end{array}$ & $\begin{array}{l}1 \\
2 \\
1 \\
- \\
- \\
-\end{array}$ & $\begin{array}{l}- \\
\overline{1} \\
- \\
- \\
-\end{array}$ \\
\hline Total & 19 & 30 & 23 & 4 & 11 & 3 & 15 & 4 & 1 & 1 & 11 & 17 & 10 & 2 & 7 & 1 & 1 & 4 & 1 \\
\hline
\end{tabular}

\section{$\mathrm{Al}=$ Ascaris lumbricoides \\ Ad $=$ Ancilostomídeos \\ $\mathrm{Tt}=$ Trichiurus trichiuro \\ Ss = Strongyloides stercoralis \\ $\mathrm{Eh}=$ Entomoeba histolytica}

por um maior espaço de tempo no sangue periférico.

Não foi possível detectar uma história antiga, pregressa, correta da população NaḍëbMaku, relacionada à malária. Contudo, pelas informações obtidas parece não ter havido esta infecção antes deste surto entre estes indígenas, mesmo porque, nesta área do Estado do Amazonas, os casos de malária são raros.

Em relação ao tratamento da malária nestes indivíduos, podemos verificar (Tabela 2) que tanto o Fansidar ${ }^{\circledR}$ ou Mefloquina foram eficazes na supressão da parasitemia. No segundo dia de tratamento todos os pacientes apresentavam a negativação da parasitemia periférica. Em todos os pacientes foi feito primaquina com o intuito de quebrar o ciclo esporogônico. Isto parece ter tido efeito, pois no décimo dia de tratamento não se encontrou gametócitos no sangue periférico destes indivíduos.

Não se usou os derivados 4-aminoquinoleinas (Cloroquina) para o tratamento das
$\mathrm{Gl}=$ Giardia lamblia

$\mathrm{Ec}=$ Entamoeba coli

En = Endolimax nana

$\mathrm{Cm}=$ Chilomastix mesnili

$\mathrm{Ph}=$ Pentatrichomonas hominis

infecções por $P$. falciparum uma vez ser sabido a ampla distribuição geográfica na Amazônia de cepas desta espécie resistentes a essa droga $^{5,17}$. Utilizou-se Fansidar ${ }^{\circledR}$ que, embora sejam conhecidas cepas de $P$. falciparum refratária a esse tratamento na Amazônia ${ }^{6}$ e assim como em outras áreas ${ }^{16}$, ele é ainda considerado um dos melhores antimaláricos para infecções por $P$. falciparum resistentes à cloroquina ${ }^{14}$.

Por ter se esgotado o Fansidar e ter-se únicamente Mefloquina, utilizou-se essa droga em doses baixas, uma vez que se conhece a eficácia deste fármaco no tratamento da malaria falciparum ${ }^{3,10}$. Esta parece ter sido a primeira vez que se utiliza a Mefloquina em ameríndios. Não foram observados efeitos colaterais e a parasitemia periférica negativou-se em dois dias.

A parasitemia periférica do paciente que recebeu Fansidar intramuscular comportou-se, praticamente, de maneira idêntica aos que receberam a droga oralmente.

Neste surto epidêmico a infecção malári 
GENARO, O. \& FERRARONI, J. J. Estudo sobre a malária e parasitoses intestinais em indígenas da tribo Nadëb-Maku, Estado do Amazonas, Brasil. Rev. Saúde públ., S. Paulo, 18:162 - 9, 1984.

ca foi mais prevalente na população jovem $(54,9 \%)$ e principalmente no sexo masculino (Tabela 1). Este é um indicador de que houve a transmissão da malária na aldeia uma vez que, pela própria estrutura social da tribo, não se deslocam da aldeia com a mesma freqüência que os adultos. Em dois ín. dios foram encontrados apenas gametócitos. $O$ tempo entre o período pré-patente e o aparecimento de gametócitos é de cerca de 7 a 12 dias para $P$. falciparum ${ }^{13}$, e é possí. vel que ambos os pacientes sejam remanescentes dos primeiros indígenas infectados e que sobreviveram à fase aguda do ataque malárico.

Durante o surto epidêmico na aldeia só se conseguiu coletar $A$. mediopunctatus. É difícil afirmar que este anofelino tenha sido o vetor responsável pela epidemia. Contudo, é uma hipótese bastante provável, mesmo porque, ele apresenta uma acentuada antropofilia, sendo o suspeito de ser transmissor da malária em algumas áreas. Ainda mais, esse anofelino foi encontrado naturalmente infectado em Narinõ e Valle del Cauca, Colôm$\mathrm{bia}^{8}$. O A. mediopunctatus é encontrado em vários países da América do $\mathrm{Sul}^{9}$, especialmente na Amazônia ${ }^{2}$. Esta epidemia teve alguns aspectos semelhantes a uma que ocorreu entre os índios de Roraima ${ }^{4}$ onde encontrou-se prevalência de $39 \%$ de infecção. Contudo, na aldeia de Roraima foi coletado $A$. darlingi e houve a ocorrência das duas espécies, $P$. falciparum e $P$. vivax.

Alguns indivíduos mostraram aparente grau de desnutrição e elevado índice de cáries dentárias. A população está relativamen. te aculturada. Diarréia foi uma afecção observada nessa população, principalmente no grupo etário mais jovem e pode não estar somente associada à infecção por protozoá- rios, mas também alimentar, com o uso de alimentos recentemente introduzidos pela cultura branca, como foi observado.

$\mathrm{O}$ alto grau de infestação por helmintos e protozoários (100\%) e de poliparasitismo traz em evidência o baixo nível de saúde desta tribo. Houve indígenas parasitados por até 7 espécies de parasitos. Cerca de $32,6 \%$ da população estudada estava parasitada por 3 espécies de parasitos. Esta alta frequiência pode ser explicada pelas péssimas condições de higiena em que vivem. As necessidades fisiológicas são executadas ao ar livre e sempre próximo às malocas. Esses resultados estão em concordância com estudos realizados em populações indígenas da Amazônia. Neel e col. $^{12}$ (1968) encontraram $96,7 \%$ de ancilos tomídeos entre os índios Xavantes. Praticamente as mesmas percentagens foram verificadas por Lawrence e col. ${ }^{P_{1}}$ (1980) em várias populações ameríndias da Amazônia, inclusive com alto grau de poliparasitismo. E interessante notar que em populações não indígenas do interior da Amazônia, a taxa de prevalência para ancilostomídeos são mais baixas. Costa ${ }^{1}$ (1947) encontrou prevalência de $37 \%$ para ancilostomídeos, em quatro cidades, enquanto Ferraroni e col. ${ }^{7}$ (1979) encontraram $30 \%$ de prevalência para o mesmo parasita na cidade de Nova Olinda do Norte, Amazonas.

\section{AGRADECIMENTOS}

A Mary E. H. Weir, Jorge R. Arias e Robert Landford pelo apoio logístico e à FU. NAI pela permissão de visitar a tribo. 
GENARO, O. \& FERRARONI, J. J. Estudo sobre a malária e parasitoses intestinais em indígenas da tribo Nadëb-Maku, Estado do Amazonas, Brasil. Rev. Saúde públ., S. Paulo, 18:162 - 9, 1984.

GENARO, O. \& FERRARONI, J. J. [A study of malaria and intestinal parasites among the Nadëb-Maku Indians of Amazonas State-Brazil]. Rev. Saúde públ., S. Paulo, 18: 162.9, 1984.

ABSTRACT: In March, 1983, a falciparum malaria outbreak occurred in the NadebMaku Indian tribe in Amazonas State, Brazil. Seventy six blood samples were obtained and examined for hematozoa with 27 (35.5\%) positive for Plasmodtum falciparum. Malaria infections were treated with Fansidar (Pyrimethamine plus sulfadoxine), mefloquine and/or primaquine. The only Anopheles species collected in the Indian tribe during the outbreak period was Anopheles mediopunctatus. All 49 stool samples obtained from the Indians were positive when examined for intestinal parasites.

UNITERMS: Plasmodium falciparum. Malaria, treatment. Indians, Brazil.

\section{REFERÊNCIAS BIBLIOGRÁFICAS}

1. COSTA, O.R. Incidência de parasitos intestinais em quatro cidades da Amazônia. Rev. Serv. esp. Saúde públ., 1: 203-19, 1947.

2.DEANE, L.M.;CAUSEY, O.R. \&DEANE, M.P. Notas sobre a distribuição e a biologia dos anofelinos das regiōes nordestinas e amazônicas do Brazil. Rev. Serv. esp. Saúde públ., 1: 827-965, 1948.

3. DOBERSTYN, E.B.; PHINTUYOTHIN, P. NOEYPATIMANLONDH, S. \& TEERAKIASTKAMJORN, C. Single-dose therapy of falciparum malaria with mefloquine or pyrimethamine-sulfadoxine. Bull. Wld Hith Org., 57: 275-9, 1979.

4. FERRARONI, J.J. \& HAYES, J. Estudo sobre um surto de malária entre os índios Mayongong e Sanomã (Norte de Rorajma). Acta amazon., 7: 401-6, 1977.

5. FERRARONI, J.J.; SPEER, C.A.; HAYES, J. \& SUZUKI, M. Prevalence of chloroquine-resistant falciparum malaria in the Brazilian Amazon. Amer. J. trop. Med. Hyg., 30: 526-30, 1981.

6. FERRARONI, J.J.; ALENCAR, F.H. \&SHRIMPTON, $R$. Multiple drug resistance in falciparum malaria from Brazil. Trans, roy. Soc. trop. Med. Hyg., 77: 138-9, 1983.

7. FERRARONI, M.J.; MONTORIL FILHO, M. \& FERRARONI, J.J.. Parasitas intestinais numa população humana da cidade de Nova Olinda do Norte, Amazonas. Acta amazon., 9:657-9, 1979.
8. FERRER, F.H. Vetores da malaria en Colombia. São Paulo, Faculdade de Higiene e Saúde Pública USP, 1956. [Curso de Entomologia Médica]

9. FORATTINI, O.P. Entomologia médica. São Paulo, Faculdade de Higiene e Saúde Pública, 1962. v. 1

10. JIANG, J.; GUO, X.; LI, G.; KONG, Y.C. \&ARNOLD, K. Antimalarial activity of Mefloquine and ginghaosu. Lancet, $2: 285.8$, 1982.

11. LAWRENCE, D.N.; NEEL, J.V.; ABADIE, S.H.; MOORE, L.L.; ADAMS, L.J.; HEALY, G.R. \& KAGAN, I.G. Epidemiologic studies among Amerindian populations of Amazonian. III. Intestinal parasitoses in newly contacted and acculturating villages. Amer. J. trop. Med. Hyg., 29: 530-7, 1980.

12 NEEL, J.V.; MIKKELSEN, W.M.; RUCKNAGEL, D.L.; WEINSTEIN, E.D.; GOYER, R.A. \& ABADIE, S.H. Further studies of the Xavante Indians. VIII. Some observations on blood, urine, and stool sam. ples. Amer. J. trop. Med. Hyg., 17: 474 85,1968 .

13. PESSÔA, S.B. \& MARTINS, A.V. Parasitologia médica. 10 å ed. Rio de Janeiro, Guanabara Koogan, 1977.

14.PETERS, W. Chemotherapy of malaria. In: Kreier, J.P., ed. Malaria. New York, Academic Press, 1980. v. 1, p. 145-285. 
GENARO, O. \& FERRARONI, J. J. Estudo sobre a malária e parasitoses intestinais em indígenas da tribo Nadëb-Maku, Estado do Amazonas, Brasil. Rev. Saúde públ., S. Paulo, 18:162 - 9, 1984.

15. PREVENTION of malaria in travellers. Morb. Mort. Wkly Rep., 31(15) 1982.

16. RUMANS, L.M.; DENNIS, D.T. \& ATMOSOEDJONOS, S. Fansidar-resistant falciparum malaria in Indonesia. Lancet, 1: 580-1, 1979.
17. WORID HEALTH ORGANIZATION. Scientific Group on Chemotherapy of Malaria and Resistance to Antimalarials, Geneva, 1972. Report. Geneva, 1973. (Techn. Rep. Ser., 529).

Recebido para publicaçd̄o em 13/06/1983. Aprovado para publicação em 24/12/1983. 